\title{
A Case of Primary Cardiac Sarcoma
}

\author{
Omair M Ali' ${ }^{1}$ Quratulanne Sheheryar ${ }^{1,2 *}$ and Yassar Nabeel ${ }^{1,2,3}$ \\ ${ }^{1}$ Wright State University, Dept. of Cardiology \\ ${ }^{2}$ Khyber Teaching Hospital, Peshawar, Pakistan, Khyber Medical University \\ ${ }^{3}$ University of Iowa Hospitals and Clinics, Iowa City, Iowa
}

Received: December 10, 2013; Accepted: May 23, 2014, Published: May 25, 2014

*Corresponding author: Quratulanne Sheheryar MD, Khyber Teaching Hospital, Peshawar, Pakistan, E-mail: Quratulanne.sheheryar@gmail.com

\begin{abstract}
Primary cardiac sarcomas are extremely rare tumors of the heart with a very poor prognosis. The treatment of choice of these rare tumors is complete excision of the tumor but it is dependent upon the stage and the extent of infiltration at presentation. We present to you two cases of Primary Cardiac Sarcoma with features of a presumed pulmonary embolism which was ruled out by investigations but did reveal a large pericardial effusion and disturbed contours of the heart in Case 1. The initial differential diagnoses included Cardiac Sarcoma, Malignant Sarcoma, Lymphoma and melanoma; but cardiac biopsy proved that it was a Cardiac Sarcoma. In our second case we present an old man with initial presenting symptoms of a worsening flu shortness of breath and shoulder pain later confirmed after investigations as Primary cardiac sarcoma. The patients were treated by excision of the tumor. Currently aggressive treatment with complete excision of the Primary Cardiac Sarcomas seems to be the best available treatment option for an otherwise fatal disease.
\end{abstract}

\section{Introduction}

Cardiac Sarcoma is a very rare kind of tumor. Primary Cardiac Sarcomas are malignant tumors which originate in the heart [1]. They have an incidence of 0.0017 to $0.0190 \%$. Among all cardiac tumors $75 \%$ are myxomas (benign) whereas $25 \%$ are malignant cardiac tumors [2]. Among the malignant cardiac tumors, 75\% are cardiac sarcomas. The right side of the heart is usually affected by these rare malignant tumors. Hemangiosarcoma is the most common type of sarcoma usually affecting the right atrium. The timely diagnosis of Primary Cardiac Sarcomas is usually overlooked because of their rare occurrence and the non-specific signs and symptoms. The most usual symptoms of Cardiac Sarcomas are chest pain, fatigue, dyspnea, infarction of limbs (usually due to embolisms from the left side of the heart), stroke and metastasis to different parts of the body. Tumors with deep infiltration can cause Superior Vena Cava Syndrome and Dysphonia because of recurrent laryngeal nerve palsy [3]. Signs including hypotension, malaise, tachycardia, diminished heart sounds and a friction rub may be present. Intractable cardiac failure can result from cardiac tamponade. If the myocardium gets involved it can result in arrhythmias, angina, heart block, heart failure, infarction, valvular obstruction or valvular insufficiency. Primary Cardiac Sarcomas include Rhabdomyosarcoma,
Mesothelioma, Angiosarcoma, Fibrosarcoma and Malignant Schwannoma [4].

The management and treatment of the tumors depends mainly upon the size of the tumor, its location and extent of metastasis. If symptoms begin to appear it means that most likely the tumor has spread to such an extent that treatment is going to be highly challenging. An open heart surgical excision of the tumor can be done if the tumor is localized. In cases where excision cannot be done due to advancement of the stage of tumor, Cardiac Transplantation is an option but after transplantation immunosuppressive drugs have to be administered so that the body does not reject foreign tissue. A lethal side-effect of these immune-suppressants is that they stimulate new growth of the cardiac sarcoma. One of the latest treatment options is autotransplantation in which the heart is removed, the sarcoma is excised and the heart is transplanted back, since it is not foreign to the body immune-suppressants do not have to be given to the patient [5].

\section{Case Presentation (Description)}

\section{Case 1}

A 50 year old female with a history of hypothyroidism, presented with progressive shortness of breath on exertion and worsening lower extremity edema. She also described intermittent sub-sternalchest pressure, also worsening with exertion. She otherwise denied paroxysmal nocturnal dyspnea, orthopnea, palpitations or weight gain. Her vitals were stable. Cardiac auscultation was for regular rate and rhythm and normal heart sounds. There was absence of jugular venous distension. Examination was otherwise within normal limits.

Comprehensive metabolic panel and complete blood count was within normal limits.

The initial concern was for pulmonary embolism. A CT of the chest did not reveal embolism but did show a large pericardial effusion and noted for abnormal configuration of the left ventricular wall concerning for a mass.

A stat echocardiogram revealed normal right and left ventricular size and function, with normal appearing valve leaflets. There was concern for massive circumferential 
pericardial effusion. There was no conclusive evidence of tamponade physiology based on echocardiographic findings. There appeared to be a loculated exudate attached to the right ventricular surface. The free wall of the rightatrium appeared to have diastolic indentation.

Cardiology team was consulted. A right heart catheterization was done. The fluid was sent for cell count and differential, LDH and protein. Hemodynamics revealed right atrial pressure (RAP) of 24/22 mm Hg (systolic/diastolic), pericardial pressure (PP) of $27 \mathrm{~mm} \mathrm{Hg}$, right ventricular pressure (RVP) of $41 / 20 \mathrm{~mm} \mathrm{Hg}$ (systolic/diastolic), pulmonary artery pressure (PAP) of 39/24 $\mathrm{mm} \mathrm{Hg}$ (systolic/diastolic) and mean of $31 \mathrm{~mm} \mathrm{Hg}$, pulmonary artery wedge pressure (PCWP) of $37 / 30 \mathrm{~mm} \mathrm{Hg}$ (systolic/ diastolic) and $31 \mathrm{~mm} \mathrm{Hg}$. A pericardiocentesis was then done. The post pericardiocentesis RAP was $18 / 18$ and PP was $18 / 2 \mathrm{~mm} \mathrm{Hg}$ with good respirophasic changes. The fluid itself was a large and bloody effusion. It was sent for gram stainand cultures including viral, fungal and AFB. These returned negative. It was also sent for cytology. Meanwhile the patient's symptoms improved. She was transferred to the CICU with a pericardial drain.

Cardiothoracic surgery team was asked to evaluate the cardiac mass. A cardiac MRI was performed (Figure $1 \mathrm{~A}-\mathrm{C}$ ). This revealed a large infiltrative mass measuring about $9.1 \mathrm{~cm}$ craniocaudually, $6.5 \mathrm{~cm}$ transversely, and $6 \mathrm{~cm}$ in anteroposterior diameter. Enhancement was demonstrated. The mass involved the posterolateralwall of the left atrium and a portion of the left ventricle. The mass extended superiorly and partially surrounded the pulmonary veins on the left. There were several nodular components of the mass that were protruding into the lumen of the left atrium. There was involvement of the mitral valve.
Based on this data, our differentials included cardiac sarcoma as well as metastatic sarcoma or melanoma, and lymphoma.

Cytology returned for reactive mesothelial cells. Cardiac biopsy was pursued to illicit pathology. This returned for primary cardiac sarcoma. The patient was transferred to Cleveland Clinic, Ohio for further management including oncology evaluation.

\section{Case 2}

A 62 year old male presented for evaluation of one month history of progressive cold, headache, and general malaise. This was complicated with recent development of new shortness of breath. Further history revealed left shoulder pain. He asked to be treated for his ongoing "Flu". He had undergone numerous treatments under his primary care's guidance including antibiotics. Vital signs were for a blood pressure of 88/72 mm $\mathrm{Hg}$ and heart rate of 93 beats per minute. Cardiopulmonary exam revealed distant heart sounds. Chest radiograph revealed an enlarged cardiac silhouette. EKG revealed low voltage QRS. An echocardiogram done in the emergency room revealed a large pericardial effusion tamponade physiology.

Cardiology team and Cardiothoracic surgery teams were in agreement that emergent pericardiocentesis be performed. This returned $900 \mathrm{cc}$ of sanguinous fluid. The fluid was sent for cell count and differential, LDH, protein, gram stain and cultures (viral, fungal and AFB). These studies returned normal. A pericardial drain was established. A trans-esophageal echocardiogram (Figure 2) showed a $3.8 \times 3.5 \mathrm{~cm}$ right atrial mass with mobile components. There appeared to be transmural extension. Based on site of origin andtransmural extension, initial differentials included metastasis or sarcomatous malignant primary lesion
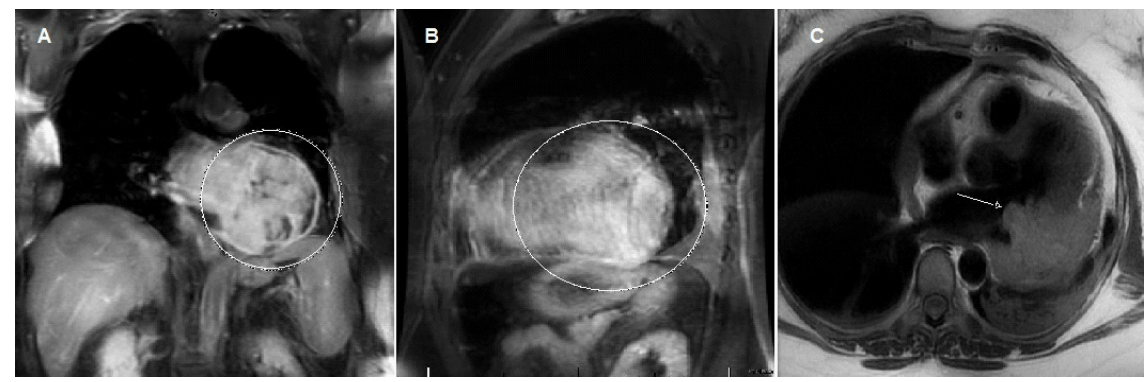

Figure 1: 1A, 1B, 1C: Intra-cardiac mass involving the posterior lateral wall of the left atrium and a portion of the left ventricle.
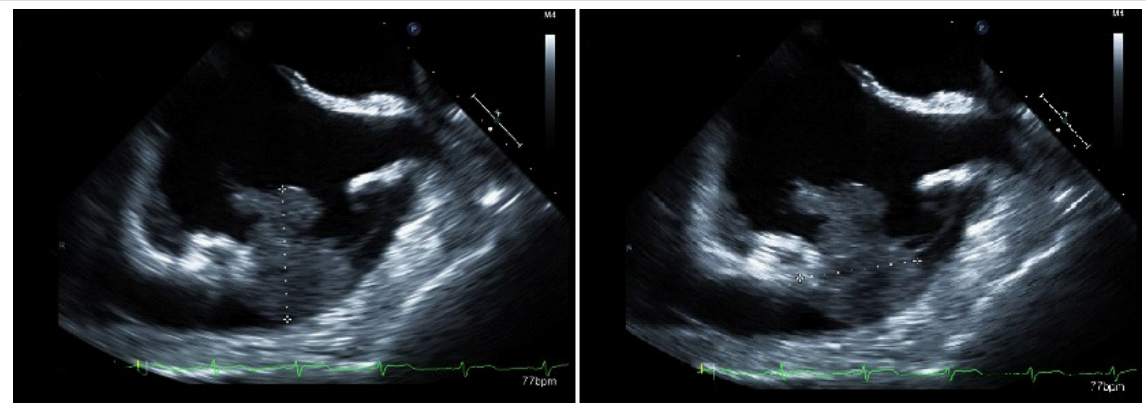

Figure 2: Transesophageal echocardiogram with intra-cardiac mass in the left atrium. 
of the heart. He was transferred to Ohio State University for further oncological management. There, he underwent surgery with resection. Biopsy results returned for angiosarcoma. He was started on Gemzar and Taxotere.

\section{Discussion}

Cardiac Sarcomas are rare malignant tumors of the heart. These primary cardiac malignant lesions have a dismal prognosis despite the enhanced diagnostic ability of newer technology. The reasons for the dismal prognosis are advanced tumor stage at presentation, unclear and non-specific symptomatology, lack of sufficient awareness due to the rarity and delayed/misdiagnosis which results in advanced tumor stage at presentation [6].

Most frequent types of cardiac sarcomas include angiosarcomas, rhabdomyosarcoma, fibrosarcoma, undifferentiated sarcomas, leiomyosarcomas, liposarcomas and synovial sarcomas. Angiosarcoma is the most common type, usually arises in the right atrium [7, 8] and frequently presents with pulmonary and or distant metastases [9]. Undifferentiated sarcoma is another common type that predominantly affects the left atrium ${ }^{7}$, typically presenting with locally advanced tumour [9].

Cardiac sarcomas are usually asymptomatic until an advanced stage. When symptomatic, they present atypically because of non-specificity. Clinical manifestations occur due to different mechanisms including obstruction to blood flow through the heart, valvular dysfunction, local invasion causing arrhythmia or pericardial effusion with/without tamponade, tumor embolism and systemic involvement $[6,10]$. The patients can complain of dyspnea, chest pain and/or generalized fatigue. No distinct physical features can be discovered on examination. Usually an audible plop can be heard through the mitral valve due to tumor prolapse. Embolization on the left side can lead to cerebrovascular accident and peripheral organ infarction. Compression effects on the larynx may cause dysphonia and on the superior vena cava may cause superior venal cava syndrome.

Echocardiography, computed tomography and MRI are the most common diagnostic and staging modalities used to delineate tumor presence and extension. Echocardiogram is mostly the initial imaging modality for cardiac tumors with Trans-esophageal Echocardiogram providing more detailed information about tumor location and extent compared with Trans-thoracic Echocardiogram [11-13, 18]. CT scan provides detailed information about the morphology, location and extent of mass and is very useful for assessment of extra cardiac disease and metastasis [14, 11, 15]. Cardiac MRI provides precise information about the location and involvement of myocardial as well as mediastinal structures $[16,17,19]$. CT scan is useful for assessing extra cardiac extension while MRI is better for evaluation of myocardial and great vessels invasion. Tumor biopsy is the gold standard for tissue diagnosis after detection of cardiac tumor with imaging modalities. While the mentioned imaging techniques in isolation are helpful in making a diagnosis, using them is conjunction with each other was most helpful in making our diagnosis. As with the cases above, definitive diagnosis comes from surgical tissue or cytological biopsy.

Patient's survival strictly depends upon the stage of the disease and the possibility of complete surgical excision. Survival rates are better with complete resection of the tumor compared to incomplete resection and metastatic disease [20]. In a series of 34 cardiac sarcoma patients, median survival period of patients with metastatic disease was 5 months, compared with 15 months in patients without metastatic disease [21]. Some case reports have documented survival up to 25 months in patients with early disease [22]. High grade sarcomas have been associated with the worse prognosis [23]. Most of the patients ultimately die of metastasis or heart failure [24].

Complete surgical resection has been reported to improve survival the most and whenever possible, is the treatment of choice $[25,26]$. In a case series, patients who underwent surgery had a median survival of 12 months whereas those who did not undergo surgery had a median survival of 1 month [25]. Surgery also has a role in palliative resections for relief of symptoms and biopsy to confirm diagnosis [26].

Although complete resection is the treatment of choice, it is not an option in many cases either because of the extensive local expansion of the tumor at the time of diagnosis or because of the presence of distant metastases [27]. Complete resection of the tumor in combination with adjuvant chemoradiotherapy offers the best chance at survival [28, 29, 30]. Despite promising responses to chemotherapy in some studies treatment approaches that involve neo-adjuvant chemotherapy and radiotherapy remain purely experimental [26].

There is no general agreement on efficacy of postsurgical therapy for cardiac sarcomas. Chemotherapy after surgical resection is recommended, even when clear surgical margins are obtained because of the high probability of missed microscopic disease and likely aggressive course. [31].

Although radiotherapy has been used for treatment of positive margins after resections, palliation of aggressive localized disease and local or isolated distant recurrence, its role remains unproven and only anecdotal responses are reported [25, 31, 32].

In selected patients without extra cardiac disease, cardiac transplantation has been reported to improve survival, with most of these patients undergoing chemotherapy and radiation therapy prior to transplantation [33-36].

Cardiac auto-transplantation which consists of explantation of heart, complete tumor excision in vivo, reconstruction of heart and then re-implantation in native position has also been reported in few case reports to prolong survival [35,36].

\section{References}

1. Reardon M, Walkes J, DeFelice C, Wojciechowski Z (2006) Cardiac Autotransplantation for Surgical Resection of a Primary Malignant Left Ventricular Tumor. Tex Heart Inst J 33(4): 495-497.

2. Devbhandari M, Meraj S, Jones MT, Kadir I, Bridgewater B (2007) Primary cardiac sarcoma- reports of two cases and a review of current literature. Journal of Cardiothoracic Surgery 24(2):34. 
3. Steger CM, Hager T, Ruttman E (2012) Primary cardiac tumours: a singlecenter 41-Year Experience. ISRN Cardiology Article ID 906109, 1-7.

4. Raaf J, Konstantakos A, Raaf H (2013) Cardiac Sarcoma Clinical Presentation. Medscape.

5. Lindenfeld J, Miller G, Shakar S, Zolty R, Lowes B et al.(2004) Drug Therapy in the heart Transplant Recipient Part II Immunosuppresive drugs- Circulation 110: 3858-3865

6. Shanmugam G. (2006) Primary Cardiac Sarcoma European Journal of Cardiothoracic Surgery $29:$ 925-932.

7. Kim CH, Dancer JY, Coffey D, Zhai QJ, Reardon M, et al. (2008) Clinicopathologic study of 24 patients with primary cardiac sarcomas: a 10-year single institution experience. Hum Pathol Jun 39(6):933-8 doi: 10.1016/j.humpath.2007.12.018.

8. Putnam JB Jr, Sweeney MS, Colon R, Lanza LA, Frazier OH, et al. (1991) Primary cardiac sarcomas. Source Department of Thoracic Surgery, University of Texas M.D. Anderson Cancer Center, Houston 77030-4009. Ann Thorac Surg Jun 51(6):906-10.

9. Shapira OM, Korach A, Izhar U, Koler T, Wald O, Ayman M, et al (2013) Radical multidisciplinary approach to primary cardiac sarcomas. Eur J Cardiothorac Surg Aug 44(2):330-510.

10. Meng Q, Lai H, Lima J, Tong W, Qian Y et al. (2002) Echocardiographic and pathologic characteristics of primary cardiac tumors: a study of 149 cases. Int J Cardiol 84: 69-75.

11. Auger D, Pressacco J, Marcotte F, Tremblay A, Dore A, et al.(2011) Cardiac masses: an integrative approach using echocardiography and other imaging modalities. Heart 97(13):1101-9.

12. Alam M, Rosman HS, Grullon C (1995) Transesophageal echocardiography in evaluation of atrial masses. Angiology 46(2):123-8.

13. Lee EY, Jeon ES, Choi JO, Lee SC, Park SW et al. (2011) Primary cardiac sarcoma mimicking mural thrombus. Eur J Echocardiogr 12(9):713.

14. Dawson WB, Mayo JR, Müller NL (1900) Computed tomography of cardiac and pericardial tumors. Can Assoc Radiol J 41(5):270-5.

15. Hoey E, Ganeshan A, Nader K, Randhawa K, Watkin R (2012) Cardiac neoplasms and pseudotumors: imaging findings on multidetector CT angiography. Diagn Interv Radiol 18(1):67-77.

16. Freedberg RS, Kronzon I, Rumancik WM, Liebeskind D(1988) The contribution of magnetic resonance imaging to the evaluation of intracardiac tumors diagnosed by echocardiography. Circulation 77(1):96-103.

17. Randhawa K, Ganeshan A, Hoey ET. Magnetic resonance imaging of cardiac tumors: part 2, malignant tumors and tumor-like conditions. Curr Probl Diagn Radiol 40(4):169-79.

18. Debourdeau P, Gligorov J, Teixeira L, Aletti M, Zammit C (2004) Malignant cardiac tumors. Bull Cancer 91(3):136-146

19. Kim CH, Dancer JY, Coffey D, Zhai QJ, Reardon M, et al. (2008) Clinicopathologic study of 24 patients with primary cardiac sarcomas: a 10-year single institution experience. Hum Pathol 39(6):933-938.

20. Fatima J, Duncan AA, Maleszewski JJ, Kalra M, Oderich GS et al.(2012) Primary angiosarcoma of the aorta, great vessels, and the heart. J Vasc Surg 2013 Mar 57(3):756-64.

21. Simpson L, Kumar SK, Okuno SH, Schaff HV, Porrata LF et al. (2008) Malignant primary cardiac tumors: review of a single institution experience. Cancer 112(11):2440-6.

22. HN. Raaf, JH. Raaf (1994) Sarcomas related to the heart and vasculature. Semin Surg Oncol 10(5) 374-382

23. Hamidi M, Moody JS, Weigel TL, Kozak KR (2010) Primary cardiac sarcoma. Ann Thorac Surg Jul 90(1):176-81.

24. Devbhandari MP, Meraj S, Jones MT, Kadir I, Bridgewater B. (2007) Primary cardiac sarcoma: reports of two cases and a review of current literature. J Cardiothorac Surg. Jul 24;2:34

25. Centofanti P, Di Rosa E, Deorsola L, Dato GM, Patane F, et al. (1999) Primary cardiac tumors: early and late results of surgical treatment in 91 patients. Ann ThoracSurg; 68(4):1236-1241.

26. Mayer F, Aebert H, Rudert M, Konigsrainer A, Horger M et al. (2007) Primary malignant sarcomas of the heart and great vessels in adult patients-a single-center experience. Oncologist; 12(9): 1134-1142.

27. Butany J, Nair V, Naseemuddin A, Nair GM, Catton C etal.(2001) Cardiac tumours: diagnosis and management. Heart; 85(2): 218-222.

28. 28. Yokouchi Y, Hiruta N, Oharaseki T, Ihara F, Oda Y etal. (2011) Primary cardiac synovial sarcoma: a case report and literature review. Pathol Int; 61(3): 150-155.

29. Reardon MJ, Walkes JC, Benjamin R (2006) Therapy insight: malignant primary cardiac tumors. Nat Clin Pract Cardiovasc Med; 3(10): 548-553.

30. Movsas B, Teruya-Feldstein J, Smith J, Glastein E, Epstein AH (1998) Primary cardiac sarcoma: a novel treatment approach. Chest; 114(2): 648-652.

31. Pessotto R, Silvestre G, Luciani GB, Anselmi M, Pasini F et al. (1997) Primary cardiac leiomyosarcoma: seven-year survival with combined surgical and adjuvant therapy. Int J Cardiol; 60(1): 91-94.

32. Gowdamarajan A, Michler RE. (2000) Therapy for primary cardiac tumors: is there a role for heart transplantation? CurrOpinCardiol;15(2): 121-125.

33. Uberfuhr P, Meiser B, Fuchs A, Schulze C, Reichenspurner H et al. (2002) Heart transplantation: an approach to treating primary cardiac sarcoma? J Heart Lung Transplant; 21(10): 1135-1139.

34. Agaimy A, Rösch J, Weyand M, Strecker T. (2012) Primary and metastatic cardiac sarcomas: a 12-year experience at a German heart center. Int J Clin Exp Pathol; 5(9): 928-938.

35. Reardon MJ, Malaisrie SC, Walkes JC, Vaporciyan AA, Rice DC etal. (2006) Cardiac autotransplantation for primary cardiac tumors. Ann Thorac Surg; 82(2): 645-650.

36. Look Hong NJ, Pandalai PK, Hornick JL, Shekar PS, Harmon DC et al. (2012) Cardiac angiosarcoma management and outcomes: 20-year singleinstitution experience. Ann Surg Oncol; 19(8): 2707-2715 